[Chem. Pharm. Bull.

$[35$ ( 6 )2219-2227(1987) $]$

\title{
Highly Stereoselective Total Synthesis of Tylonolide, the Aglycon of the 16-Membered Macrolide Antibiotic Tylosin. II. Total Synthesis of Tylonolide by Virtue of 4-Methoxybenzyl and 3,4-Dimethoxybenzyl Protection ${ }^{1,2)}$
}

\author{
Tatsuyoshi Tanaka, Yuji Oikawa, Tatsuo Hamada, \\ and OSAMU Yonemitsu* \\ Faculty of Pharmaceutical Sciences, Hokkaido University, \\ Kita-12, Nishi-6, Kita-ku, Sapporo 060, Japan
}

(Received September 19, 1986)

\begin{abstract}
Tylonolide, was synthesized from D-glucose via coupling and cyclization of two segments i (2) $(\mathrm{C}-11-\mathrm{C}-17)$ and ii $(3)(\mathrm{C}-1-\mathrm{C}-10)$, which were synthesized from diacetoneglucose. A PrelogDjerassi lactone-type compound was an intermediate in the synthesis of the latter segment. Esterification of the two segments by Yamaguchi's method followed by macrocyclization by use of the Wittig-Horner reaction gave the 16-membered cyclic enone, whose protecting groups were removed to afford tylonolide. In this total synthesis, 4-methoxybenzyl and 3,4-dimethoxybenzyl protecting groups played an important role.
\end{abstract}

Keywords - macrolide antibiotic; tylosin; aglycon; tylonolide; esterification; macrocyclization; Wittig-Horner reaction; protecting group; 2,3-dichloro-5,6-dicyanobenzoquinone oxidation; stereoselective synthesis

For the purpose of the highly selective total synthesis of complex natural products such as macrolide and polyether antibiotics, it is essential to apply the most suitable protection for many functional groups as well as to use highly regio- and stereo-controlled reactions. As a part of our recent synthetic studies on such complex natural products, we have attempted the highly stereoselective total synthesis of tylonolide $(\mathbf{1})^{3)}$ from D-glucose by means of our new synthetic methodology recently established in the synthesis of methynolide. ${ }^{4)}$

Segments i (2) and ii (3) seemed to be the most suitable intermediate in our general strategy and, in the preceding paper, ${ }^{1)}$ the synthesis of the chiral synthon (12) was reported. In the present paper, we describe a new total synthesis of $\mathbf{1}$ through syntheses of $\mathbf{2}$ and $\mathbf{3}$, coupling of these two segments, and selective removal of 4-methoxybenzyl (MPM) ${ }^{5,6)}$ and

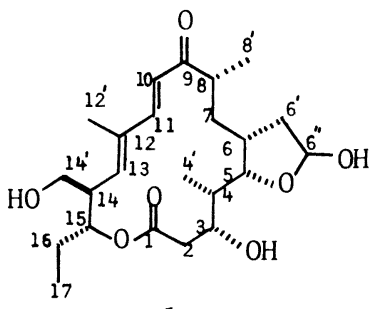

1

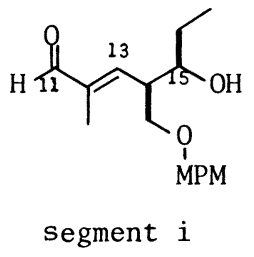

2

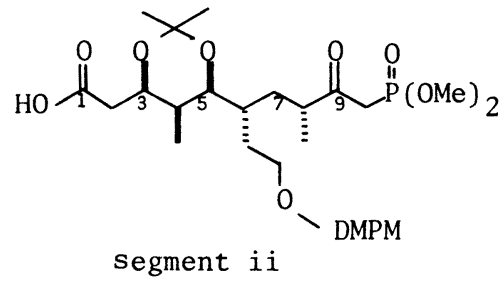

3

$\mathrm{MPM}=4-\mathrm{MeOC}_{6} \mathrm{H}_{4} \mathrm{CH}_{2}$ $\mathrm{DMPM}=3,4-(\mathrm{MeO})_{2} \mathrm{C}_{6} \mathrm{H}_{3} \mathrm{CH}_{2}$ 
3,4-dimethoxybenzyl (DMPM $)^{6,7)}$ protecting groups by the use of 2,3-dichloro-5,6dicyanobenzoquinone (DDQ) at crucial points in the final stage.

\section{Synthesis of Segment $i$}

Segment i (2), corresponding to C-11-C-17 of $1,{ }^{8)}$ was rather easily synthesized from Dglucose via the MPM ether (4). ${ }^{9,10)}$ The isopropylidene protection of the 5,6-side chain ${ }^{12)}$ was selectively removed in methanol with $2 \%$ sulfuric acid, ${ }^{13)}$ and the resulting diol was converted to the ditosylate, which was subjected to reductive elimination with sodium iodide in methyl ethyl ketone in the usual way to give the olefin (5). Catalytic reduction of 5 over $10 \%$ palladium on carbon $(\mathrm{Pd}-\mathrm{C})$ gave 6 in quantitative yield. Removal of the 1,2-isopropylidene protection $^{12)}$ in tetrahydrofuran (THF) with $4.5 \mathrm{~N}$ hydrochloric acid gave the lactol (7), which was reduced to the triol (8) by treatment with calcium borohydride ${ }^{14)}$ in high yield ${ }^{15)}$ The 1,2diol was readily cleaved with sodium metaperiodate to give the aldehyde (9) in excellent yield. When 9 was treated with a stable ylide ${ }^{16)}$ in benzene at $60^{\circ} \mathrm{C}$, a smooth Wittig reaction proceeded, and the expected $(E)-\alpha, \beta$-unsaturated ester $(\mathbf{1 0})$ was isolated in high yield. ${ }^{17,18)}$ Lithium aluminum hydride (LAH) reduction of the ester (10) gave the allyl alcohol (11), which was oxidized with manganese dioxide in dichloromethane to give the $\alpha, \beta$-unsaturated aldehyde (2) (segment i) in excellent yield.
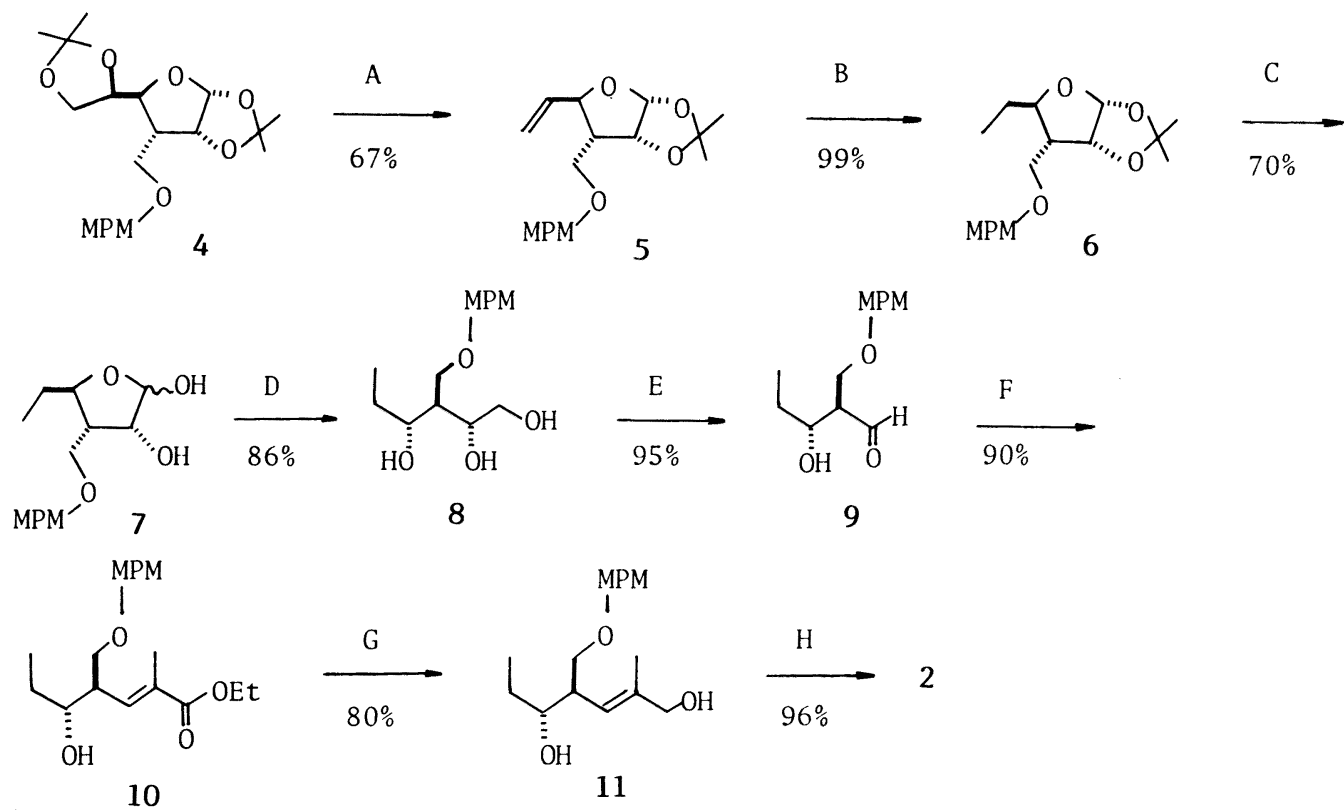

(A) 1) $2 \% \mathrm{H}_{2} \mathrm{SO}_{4}, \mathrm{MeOH}$; 2) $\mathrm{TsCl}, \mathrm{Et}_{3} \mathrm{~N}, \mathrm{DMAP}, \mathrm{CH}_{2} \mathrm{Cl}_{2}$; 3) NaI, MeCOEt, reflux (B) $\mathrm{Pd}-\mathrm{C}, \mathrm{H}_{2}$, EtOAc (C) $4.5 \mathrm{~N} \mathrm{HCl}, \mathrm{THF}, 50^{\circ} \mathrm{C}$ (D) $\mathrm{CaCl}_{2}, \mathrm{NaBH}_{4}$, EtOH (E) $\mathrm{NaIO}_{4}, \mathrm{MeOH}-\mathrm{H}_{2} \mathrm{O}$ (F) $\mathrm{Ph}_{3} \mathrm{P}=\mathrm{CMeCO}_{2} \mathrm{Et}$, benzene, 60 C (G) $\mathrm{LiAlH}_{4}, \mathrm{THF}, 0^{\circ} \mathrm{C}$ (H) $\mathrm{MnO}_{2}, \mathrm{CH}_{2} \mathrm{Cl}_{2}$

Chart 2

\section{Synthesis of Segment ii}

In the previous paper, ${ }^{1)}$ the chiral intermediate (12) having all the chiral centers required for segment ii was synthesized from D-glucose. The primary alcohol of 12 was protected with a DMPM group ${ }^{6,7)}$ to give 13 in $70 \%$ yield, ${ }^{19)}$ and then the acetal of 13 was hydrolyzed in THF with $1 \mathrm{~N}$ hydrochloric acid. The resulting lactol was reduced to the triol (14) by treatment with calcium borohydride ${ }^{14)}$ in quantitative yield. ${ }^{20)}$ The 1,3-diol group of $\mathbf{1 4}$ was protected as an 
acetonide group with 2,2-dimethoxypropane in the presence of camphorsulfonic acid (CSA) in $85 \%$ yield, and the remaining primary alcohol was subjected to Swern oxidation ${ }^{21)}$ to give the aldehyde (15) in $98 \%$ yield. The Wittig-Horner reaction of 15 with the lithio derivative of dimethyl methylphosphonate ${ }^{22)}$ gave almost quantitatively a hydroxy phosphonate, which was immediately oxidized with pyridinium dichromate $(\mathrm{PDC})^{23}$ in dimethylformamide (DMF) to give the ketophosphonate $(16) .{ }^{24)}$ Finally, among various possible oxidation methods of the terminal olefin of $\mathbf{1 6}$ into the carboxylic group, Lemieux-von Rudloff oxidation with potassium permanganate and sodium periodate ${ }^{26)}$ gave a good result, and the expected segment ii (3) was isolated in $83 \%$ yield.<smiles>C=CCC(O)C(C)[C@H]1O[C@H](OC(C)C)[C@@H](C)C[C@H]1CCO</smiles>

12

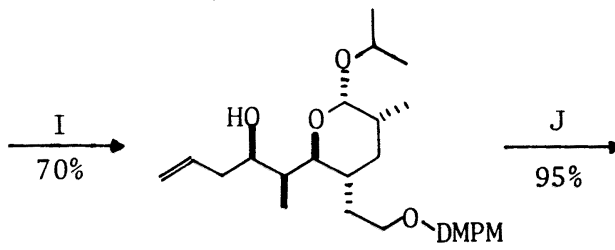

13

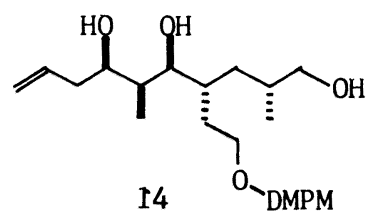

14

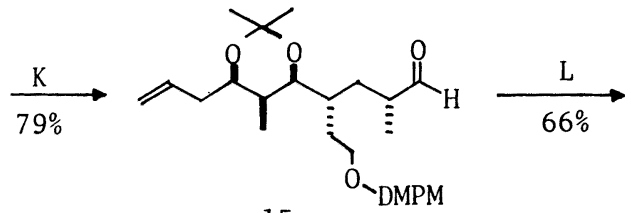
15

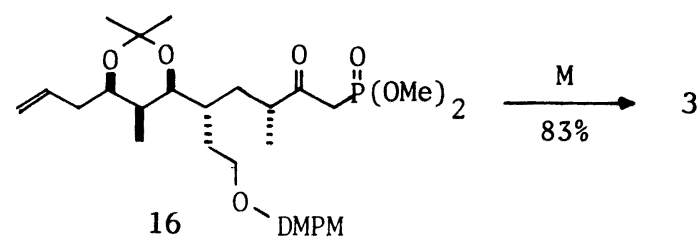

(I) $\mathrm{NaH}$, DMSO-THF, DMPMCl (J) 1 ) $1 \mathrm{~N} \mathrm{HCl}$, THF, $50^{\circ} \mathrm{C}$; 2) $\mathrm{CaCl}_{2}, \mathrm{NaBH}_{4}$, EtOH (K) 1) $(\mathrm{MeO})_{2} \mathrm{CMe}_{2}, \mathrm{CSA}$, benzene; 2) $(\mathrm{COCl})_{2}, \mathrm{DMSO}, \mathrm{CH}_{2} \mathrm{Cl}_{2}, \mathrm{Et}_{3} \mathrm{~N}$, $-80^{\circ} \mathrm{C} \rightarrow$ room temperature (L) 1) $(\mathrm{MeO})_{2} \mathrm{P}(\mathrm{O}) \mathrm{Me}, n$-BuLi, THF, $-70 \rightarrow-30^{\circ} \mathrm{C}$; 2) $\mathrm{PDC}, \mathrm{DMF}$ (M) $\mathrm{NaIO}_{4}, \mathrm{KMnO}_{4}, \mathrm{NaHCO}_{3}, \mathrm{MeCOMe}-\mathrm{H}_{2} \mathrm{O}$

Chart 3

\section{Coupling of the Two Segments and Synthesis of Tylonolide}

The macrolactonization of seco-acids is commonly used for the synthesis of macrolides. ${ }^{27)}$ Wittig-Horner coupling between the two segments i (2) and ii (3) was first examined in order to obtain a seco-acid, but all attempts were unsuccessful. Therefore, we decided to reverse the order of reactions, namely esterification between $\mathbf{2}$ and $\mathbf{3}$ was first examined, and the resulting ester was subjected to intramolecular Wittig-Horner reaction.

When 2 and 3 were coupled in a rather concentrated solution (more than $1 \mathrm{M}$ ) with dicyclohexylcarbodiimide (DCC) in the presence of 4-dimethylaminopyridine (DMAP), the expected ester (17) was obtained in $61 \%$ yield, but unfortunately complete separation from impurities could not be achieved. However, the Yamaguchi method ${ }^{27 g)}$ gave a much better result. When 2 and 3 were condensed with 2,4,6-trichlorobenzoyl chloride in the presence of triethylamine and DMAP, the esterification was completed within only $1 \mathrm{~h}$ at room temperature to give the ester (17) in $66 \%$ yield.

The intramolecular Wittig-Horner coupling of 17 was carried out under Aristoff ${ }^{28)}$ Nicolaou ${ }^{3 b)}$ conditions, namely when a $1 \mathrm{~mm}$ solution of 17 in toluene was heated at $100{ }^{\circ} \mathrm{C}$ in the presence of 18 -crown-6 (12 eq) and potassium carbonate (6 eq), the starting material (17) disappeared after $12 \mathrm{~h}$, and the expected 16 -membered enone (18) was isolated in $59 \%$ yield.

Selective removal of DMPM protecting groups in the presence of MPM protecting groups by DDQ oxidation has been clearly demonstrated in many cases. ${ }^{6,7)}$ When an ice-cold 


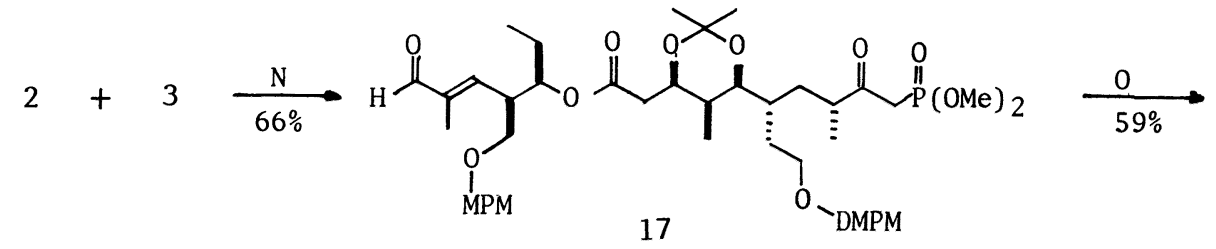

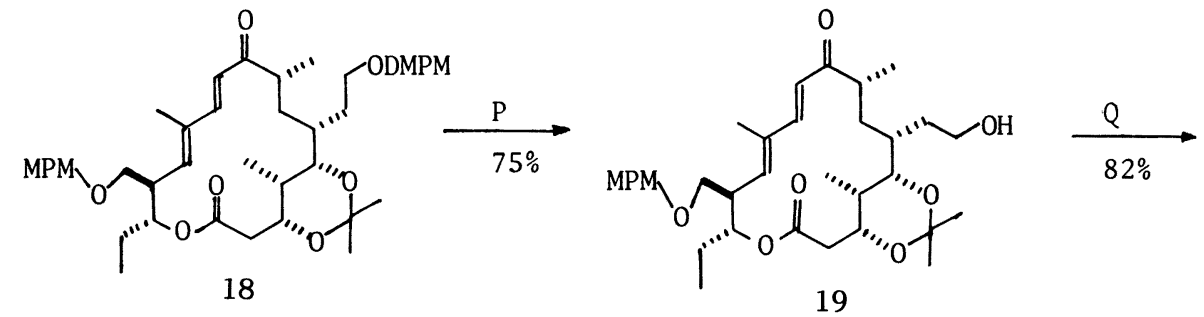<smiles>[R][C@H](C)C(C)C</smiles>

(N) 2,4,6- $\mathrm{Cl}_{3} \mathrm{C}_{6} \mathrm{H}_{2} \mathrm{COCl}, \mathrm{Et}_{3} \mathrm{~N}$, DMAP, THF (O) $\mathrm{K}_{2} \mathrm{CO}_{3}$, 18-crown-6, toluene, $100^{\circ} \mathrm{C}$ (P) DDQ, $\mathrm{CH}_{2} \mathrm{Cl}_{2}-\mathrm{H}_{2} \mathrm{O}$-benzene, $5^{\circ} \mathrm{C}$ (Q) 1) $\left.\mathrm{PDC}, \mathrm{CH}_{2} \mathrm{Cl}_{2} ; 2\right) 0.5 \mathrm{~N} \mathrm{HCl}$, THF (R) DDQ, $\mathrm{CH}_{2} \mathrm{Cl}_{2}-\mathrm{H}_{2} \mathrm{O}$

Chart 4

solution of 18 in dichloromethane-water $(20: 1)$ was treated with a slight excess of DDQ (1.2 eq), the selective deprotection of the DMPM group occurred smoothly, and the monoalcohol (19) was isolated in $75 \%$ yield. PDC oxidation ${ }^{23)}$ of the primary alcohol of 19 gave the aldehyde $(94 \%)$, and the remaining acetonide was immediately removed with $0.5 \mathrm{~N}$ hydrochloric acid to give the hemiacetal compound (20) in $90 \%$ yield. Finally, the MPM protecting group of $\mathbf{2 0}$ was easily removed by retreatment with DDQ under usual conditions, ${ }^{5,6)}$ and tylonolide (1) was isolated in $70 \%$ yield. This compound was identical, in terms of its infrared (IR), nuclear magnetic resonance (NMR) and mass spectra (MS) with tylonolide derived from natural tylosin. ${ }^{29,30)}$ Stereoselectivities in this total synthesis of 1 from D-glucose for the construction of the new chiral centers at C-3, C-4, C-6, C-8, and C-14 were $91,94,100,87$, and $100 \%$, respectively.

\section{Experimental}

Physical data were measured as described in the previous paper. ${ }^{4 a)}$

1,2-O-Isopropylidene-3- $C$-(4-methoxybenzyl)oxymethyl-3,5,6-trideoxy- $\alpha$-D-ribo-hex-5-enofuranose (5)- $\mathrm{A}$ solution of $4(3.52 \mathrm{~g}, 8.95 \mathrm{mmol})$ in $\mathrm{MeOH}(43 \mathrm{ml})$ and $2 \% \mathrm{H}_{2} \mathrm{SO}_{4}(18 \mathrm{ml})$ was allowed to stand at room temperature for $15 \mathrm{~h}$. After neutralization with $\mathrm{NaHCO}_{3}$, the reaction mixture was evaporated in vacuo, and extracted with $\mathrm{CH}_{2} \mathrm{Cl}_{2}$. The extract was washed with $\mathrm{H}_{2} \mathrm{O}$, dried over anhydrous $\mathrm{Na}_{2} \mathrm{SO}_{4}$, and evaporated in vacuo to leave an oil, which was chromatographed on a silica gel column with EtOAc-hexane $(2: 1)$ to give the diol as a colorless oil $(2.72 \mathrm{~g}$, $86.5 \%) . \mathrm{TsCl}(1.68 \mathrm{~g}), \mathrm{Et}_{3} \mathrm{~N}(2 \mathrm{~g})$ and DMAP $(50 \mathrm{mg})$ were added to a stirred $\mathrm{CH}_{2} \mathrm{Cl}_{2}$ solution $(50 \mathrm{ml})$ of the diol $(1.31 \mathrm{~g}, 3.8 \mathrm{mmol})$ at room temperature. After $19 \mathrm{~h}$, the reaction mixture was diluted with $\mathrm{CH}_{2} \mathrm{Cl}_{2}$, washed with $1 \mathrm{~N}$ $\mathrm{HCl}, 10 \% \mathrm{NaHCO}_{3}$ and $\mathrm{H}_{2} \mathrm{O}$, dried over anhydrous $\mathrm{Na}_{2} \mathrm{SO}_{4}$, and evaporated in vacuo to leave a pale yellow oil, which was dissolved in MeCOEt and heated under reflux with $\mathrm{NaI}(3.42 \mathrm{~g})$ for $24 \mathrm{~h}$. The reaction mixture was evaporated in 
vacuo to leave an oil, which was extracted with $\mathrm{CH}_{2} \mathrm{Cl}_{2}$. The extract was washed with $\mathrm{Na}_{2} \mathrm{~S}_{2} \mathrm{O}_{3}$ solution and $\mathrm{H}_{2} \mathrm{O}$, dried over anhydrous $\mathrm{Na}_{2} \mathrm{SO}_{4}$, and evaporated in vacuo to leave an oil, which was chromatographed on a silica gel column with EtOAc-hexane $(1: 3)$ to give 5 as a colorless solid $(0.91 \mathrm{~g}, 77 \%)$. Recrystallization from ether-hexane gave colorless needles, mp 54-56 C. ${ }^{1} \mathrm{H}-\mathrm{NMR}\left(\mathrm{CDCl}_{3}\right) \delta: 1.34(3 \mathrm{H}, \mathrm{s}), 1.51(3 \mathrm{H}, \mathrm{s}), 1.94-2.23(1 \mathrm{H}, \mathrm{m}), 3.40(1 \mathrm{H}$, dd, $J=9.5,5.0 \mathrm{~Hz}), 3.75(1 \mathrm{H}, \mathrm{dd}, J=9.5,9.0 \mathrm{~Hz}), 3.80(3 \mathrm{H}, \mathrm{s}), 4.20(1 \mathrm{H}, \mathrm{dd}, J=10.5,9.0 \mathrm{~Hz}), 4.46(2 \mathrm{H}, \mathrm{s}), 4.74(1 \mathrm{H}, \mathrm{t}$, $J=4.0 \mathrm{~Hz}), 5.15-5.39(2 \mathrm{H}, \mathrm{m}), 5.65-6.00(1 \mathrm{H}, \mathrm{m}), 5.83(1 \mathrm{H}, \mathrm{d}, J=4.5 \mathrm{~Hz}), 6.86(2 \mathrm{H}, \mathrm{d}, J=9.0 \mathrm{~Hz}), 7.25(2 \mathrm{H}, \mathrm{d}, J=$ $9.0 \mathrm{~Hz}$ ). MS $m / z$ (relative intensity): $320\left(\mathrm{M}^{+}, 0.2\right), 305(0.3), 262$ (3.5), 137 (41), 121 (100). Exact MS $m / z$ Calcd for $\mathrm{C}_{18} \mathrm{H}_{24} \mathrm{O}_{5}: 320.1623$. Found: 320.16241. Anal. Calcd for $\mathrm{C}_{18} \mathrm{H}_{24} \mathrm{O}_{5}: \mathrm{C}, 67.48 ; \mathrm{H}, 7.55$. Found: C, 67.33; H, 7.55.

1,2-O-Isopropylidene-3- $\boldsymbol{C}$-(4-methoxybenzyl)oxymethyl-3,5,6-trideoxy- $\boldsymbol{\alpha}$-D-ribo-hexofuranose (6) — An EtOAc solution $(30 \mathrm{ml})$ of $5(1.38 \mathrm{~g}, 4.31 \mathrm{mmol})$ was hydrogenated with $10 \% \mathrm{Pd}-\mathrm{C}(100 \mathrm{mg})$ at ordinary temperature and pressure for $30 \mathrm{~min}$. After removal of the catalyst by filtration, the filtrate was evaporated in vacuo to leave $\mathbf{6}$ as a colorless oil $(1.36 \mathrm{~g}, 98.5 \%) .{ }^{1} \mathrm{H}-\mathrm{NMR}\left(\mathrm{CDCl}_{3}\right) \delta: 0.98(3 \mathrm{H}, \mathrm{t}, J=7.0 \mathrm{~Hz}), 1.20-2.15(3 \mathrm{H}, \mathrm{m}), 1.32(3 \mathrm{H}, \mathrm{s}), 1.49(3 \mathrm{H}$, s), $3.44(1 \mathrm{H}, \mathrm{dd}, J=9.5,6.0 \mathrm{~Hz}), 3.65-3.91(2 \mathrm{H}, \mathrm{m}), 3.81(3 \mathrm{H}, \mathrm{s}), 4.47(2 \mathrm{H}, \mathrm{s}), 4.70(1 \mathrm{H}, \mathrm{t}, J=4.0 \mathrm{~Hz}), 5.79(1 \mathrm{H}, \mathrm{d}$, $J=3.5 \mathrm{~Hz}), 6.86(2 \mathrm{H}, \mathrm{d}, J=9.0 \mathrm{~Hz}), 7.25(2 \mathrm{H}, \mathrm{d}, J=9.0 \mathrm{~Hz}) .[\alpha]_{\mathrm{D}}^{15}+50^{\circ}\left(c=1.3, \mathrm{CHCl}_{3}\right) . \mathrm{MS} m / z$ (relative intensity): $307\left(\mathrm{M}^{+}-15,0.4\right), 264(2.9), 203(1.0), 138(58), 121$ (100). Exact MS $m / z$ Calcd for $\mathrm{C}_{17} \mathrm{H}_{23} \mathrm{O}_{5}\left(\mathrm{M}^{+}-15\right): 307.15452$. Found: 307.15520 .

3-C-(4-Methoxybenzyl)oxymethyl-3,5,6-trideoxy- $\boldsymbol{\alpha}$-D-ribo-hexofuranose (7)—A solution of 6 (1.63 g, 5.06 $\mathrm{mmol})$ in THF $(30 \mathrm{ml})$ and $4.5 \mathrm{~N} \mathrm{HCl}(10 \mathrm{ml})$ was heated at $50^{\circ} \mathrm{C}$ for $5.5 \mathrm{~h}$. After neutralization with $\mathrm{NaHCO}_{3}$, the reaction mixture was evaporated in vacuo, and the residue was extracted with $\mathrm{CH}_{2} \mathrm{Cl}_{2}$. The extract was dried over anhydrous $\mathrm{Na}_{2} \mathrm{SO}_{4}$ and evaporated in vacuo, and the residue was chromatographed on a silica gel column with EtOAc-hexane $(2: 1)$ to give $7(1.0 \mathrm{~g}, 70 \%)$. Recrystallization from ether-hexane gave colorless crystals, mp $61-$ $64 \mathrm{C}$. MS $m / z$ (relative intensity): $282\left(\mathrm{M}^{+}, 0.8\right), 264$ (1.2), 203 (1.2), 137 (43), 121 (100). Exact MS $m / z$ Calcd for $\mathrm{C}_{15} \mathrm{H}_{22} \mathrm{O}_{5}\left(\mathrm{M}^{+}\right): 282.14669$. Found: 282.14862. Anal. Calcd for $\mathrm{C}_{15} \mathrm{H}_{22} \mathrm{O}_{5}: \mathrm{C}, 63.80 ; \mathrm{H}, 7.85$. Found: $\mathrm{C}, 63.66 ; \mathrm{H}$, 7.81 .

$(2 R, 3 R, 4 S)$-2-(4-Methoxybenzyl)oxymethyl-1,2,4-trihydroxyhexane (8)- $-\mathrm{A}$ solution of $\mathrm{NaBH}_{4}(400 \mathrm{mg}$, $10.5 \mathrm{mmol})$ in $\mathrm{EtOH}(50 \mathrm{ml})$ was added dropwise to a cold stirred $\mathrm{EtOH}$ solution $(50 \mathrm{ml})$ of $\mathrm{CaCl}_{2}(1.1 \mathrm{~g})$ at $-20^{\circ} \mathrm{C}$ under argon. The resulting $\mathrm{Ca}\left(\mathrm{BH}_{4}\right)_{2}$ solution was stirred for $30 \mathrm{~min}$ below $-10^{\circ} \mathrm{C}$, then a solution of $7(900 \mathrm{mg}$, $3.19 \mathrm{mmol}$ ) in EtOH was added and the mixture was stirred at room temperature for $1 \mathrm{~h}$. The reducing agent was decomposed with $4 \mathrm{~N} \mathrm{HCl}$, and the solution was neutralized with $\mathrm{NaHCO}_{3}$. After removal of precipitates by filtration, the filtrate was evaporated in vacuo and the residue was extracted with $\mathrm{CH}_{2} \mathrm{Cl}_{2}$. The extract was dried and evaporated in vacuo to leave an oil, which was chromatographed on a silica gel column with $\mathrm{CH}_{2} \mathrm{Cl}_{2}-\mathrm{EtOH}(20: 1)$ to give 8 as a colorless oil $(777 \mathrm{mg}, 86 \%) .{ }^{1} \mathrm{H}-\mathrm{NMR}\left(\mathrm{CDCl}_{3}\right) \delta: 0.97(3 \mathrm{H}, \mathrm{t}, J=7 \mathrm{~Hz}), 1.30-1.70(2 \mathrm{H}, \mathrm{m}), 1.88(1 \mathrm{H}, \mathrm{dq}, J=4$, $6 \mathrm{~Hz}), 3.52(1 \mathrm{H}, \mathrm{dd}, J=5,9.5 \mathrm{~Hz}), 3.58(1 \mathrm{H}, \mathrm{dd}, J=3.5,9.5 \mathrm{~Hz}), 3.65(1 \mathrm{H}, \mathrm{dd}, J=5,11.5 \mathrm{~Hz}), 3.72(1 \mathrm{H}, \mathrm{dd}, J=4$, $11.5 \mathrm{~Hz}), 3.75-3.88(1 \mathrm{H}, \mathrm{m}), 3.81(3 \mathrm{H}, \mathrm{s}), 3.98(1 \mathrm{H}, \mathrm{dt}, J=4.5,5 \mathrm{~Hz}), 4.41(2 \mathrm{H}, \mathrm{s}), 6.88(2 \mathrm{H}, \mathrm{d}, J=9 \mathrm{~Hz}), 7.22(2 \mathrm{H}, \mathrm{d}$, $J=9 \mathrm{~Hz}$ ). MS $m / z$ (relative intensity): $284\left(\mathrm{M}^{+}, 0.5\right), 266(3.8), 178(1.8), 150(2.6), 137(44), 121(100)$. Exact MS $m / z$ Calcd for $\mathrm{C}_{15} \mathrm{H}_{24} \mathrm{O}_{5}\left(\mathrm{M}^{+}\right)$: 284.16235. Found: 284.16259. $[\alpha]_{\mathrm{D}}^{15}+7.8^{\circ}\left(c=1.3, \mathrm{CHCl}_{3}\right)$.

(2S,3R)-3-Hydroxy-2-[(4-methoxybenzyl)oxymethyl]pentanal (9) A solution of $\mathrm{NaIO}_{4}(430 \mathrm{mg}, 2.0 \mathrm{mmol})$ in $\mathrm{H}_{2} \mathrm{O}(3.5 \mathrm{ml})$ was added to a stirred $\mathrm{MeOH}$ solution $(10 \mathrm{ml})$ of $\mathbf{8}(194 \mathrm{mg}, 0.68 \mathrm{mmol})$ at room temperature. After $1 \mathrm{~h}$, the precipitate was filtered off, and the filtrate was evaporated in vacuo. The residue was taken up in $\mathrm{CH}_{2} \mathrm{Cl}_{2}$, and the solution was dried over $\mathrm{Na}_{2} \mathrm{SO}_{4}$, then evaporated in vacuo to leave an oil, which was chromatographed on a silica gel column with EtOAc-hexane $(1: 1)$ to give 9 as a colorless oil $(164 \mathrm{mg}, 95 \%) .{ }^{1} \mathrm{H}-\mathrm{NMR}\left(\mathrm{CDCl}_{3}\right) \delta: 0.99(3 \mathrm{H}, \mathrm{t}, J=$ $7.0 \mathrm{~Hz}), 1.60(2 \mathrm{H}$, quint, $J=7.0 \mathrm{~Hz}), 2.45-2.63(1 \mathrm{H}, \mathrm{m}), 2.80(1 \mathrm{H}, \mathrm{br} \mathrm{s}), 3.67-4.20(3 \mathrm{H}, \mathrm{m}), 3.81(3 \mathrm{H}, \mathrm{s}), 4.46(2 \mathrm{H}, \mathrm{s})$, $6.86(2 \mathrm{H}, \mathrm{d}, J=9.0 \mathrm{~Hz}), 7.25(2 \mathrm{H}, \mathrm{d}, J=9.0 \mathrm{~Hz}), 9.87(1 \mathrm{H}, \mathrm{d}, J=1.5 \mathrm{~Hz}) . \mathrm{MS} m / z$ (relative intensity): $252\left(\mathrm{M}^{+}, 0.4\right)$, 234 (0.4), 194 (1.8), 175 (10), 137 (92), 121 (100). Exact MS $m / z$ Calcd for $\mathrm{C}_{14} \mathrm{H}_{20} \mathrm{O}_{4}\left(\mathrm{M}^{+}\right): 252.13614$. Found: 252.13501. IR $v_{\max }^{\text {neat }} \mathrm{cm}^{-1}: 3400,1720 .[\alpha]_{\mathrm{D}}^{15}+6.9^{\circ}\left(c=1.3, \mathrm{CHCl}_{3}\right)$.

Ethyl $5(R)$-Hydroxy-4(R)-(4-methoxybenzyl)oxymethyl-2-methyl-2(E)-heptenoate (10)_A benzene solution $(20 \mathrm{ml})$ of $9(545 \mathrm{mg}, 2.16 \mathrm{mmol})$ and recrystallized 1-carboethoxyethylidenetriphenylphosphorane ${ }^{16)}(1.6 \mathrm{~g}, 4.4$ $\mathrm{mmol}$ ) was heated at $60 \mathrm{C}$ for $10 \mathrm{~h}$. After evaporation of the solvent in vacuo, the residue was chromatographed on a silica gel column with EtOAc-hexane $(1: 2)$ to give 10 as a colorless oil $(653 \mathrm{mg}, 90 \%) .{ }^{1} \mathrm{H}-\mathrm{NMR}\left(\mathrm{CDCl}_{3}\right) \delta: 0.93(3 \mathrm{H}$, $\mathrm{t}, J=7.0 \mathrm{~Hz}), 1.30(3 \mathrm{H}, \mathrm{t}, J=7.0 \mathrm{~Hz}), 1.25-1.60(2 \mathrm{H}, \mathrm{m}), 1.86(3 \mathrm{H}, \mathrm{d}, J=1.5 \mathrm{~Hz}), 2.50(1 \mathrm{H}, \mathrm{br} \mathrm{s}), 2.60-2.90(1 \mathrm{H}, \mathrm{m})$, $3.53(1 \mathrm{H}, \mathrm{dd}, J=12.0,1.0 \mathrm{~Hz}), 3.63(1 \mathrm{H}, \mathrm{dd}, J=12.0,2.5 \mathrm{~Hz}), 3.70-3.95(1 \mathrm{H}, \mathrm{m}), 3.81(3 \mathrm{H}, \mathrm{s}), 4.19(2 \mathrm{H}, \mathrm{q}, J=$ $7.0 \mathrm{~Hz}), 4.45(2 \mathrm{H}, \mathrm{s}), 6.81(1 \mathrm{H}, \mathrm{dq}, J=10.0,1.5 \mathrm{~Hz}), 6.86(2 \mathrm{H}, \mathrm{d}, J=9.0 \mathrm{~Hz}), 7.25(2 \mathrm{H}, \mathrm{d}, J=9.0 \mathrm{~Hz}) . \mathrm{IR} v_{\max }^{\text {neat }} \mathrm{cm}^{-1}$ : 3450, 1705. $[\alpha]_{\mathrm{D}}^{16.5}-17\left(c=4.4, \mathrm{CHCl}_{3}\right)$. MS $m / z$ (relative intensity): $336\left(\mathrm{M}^{+}, 0.4\right), 263(0.8), 200(10), 121(100)$ Exact MS $m / z$ Calcd for $\mathrm{C}_{19} \mathrm{H}_{28} \mathrm{O}_{5}\left(\mathrm{M}^{+}\right): 336.19364$. Found: 336.19474 .

1,5(R)-Dihydroxy-4(R)-(4-methoxybenzyl)oxymethyl-2-methyl-2(E)-heptene (11) - A THF solution of 10 (454 $\mathrm{mg}, 1.35 \mathrm{mmol})$ was added dropwise to a cold stirred suspension of $\mathrm{LiAlH}_{4}(100 \mathrm{mg}, 2.6 \mathrm{mmol})$ in $\mathrm{THF}(5 \mathrm{ml})$ under argon in an ice-bath. After $1 \mathrm{~h}$, the reaction was quenched by the addition of $\mathrm{MeOH}$, then $\mathrm{H}_{2} \mathrm{O}(0.1 \mathrm{ml}), 15 \%$ $\mathrm{NaOH}(0.1 \mathrm{ml})$ and $\mathrm{H}_{2} \mathrm{O}(0.3 \mathrm{ml})$ were added, and the mixture was stirred for $1 \mathrm{~h}$. After removal of precipitated inorganic salts by filtration, the filtrate was evaporated in vacuo and the residue was chromatographed on a silica gel column with EtOAc-hexane $(2: 1)$ to give $\mathbf{1 1}$ as a colorless solid $(339 \mathrm{mg}, 86 \%)$. Recrystallization from benzene- 
hexane gave colorless needles, mp $78-80 \mathrm{C} .{ }^{1} \mathrm{H}-\mathrm{NMR}\left(\mathrm{CDCl}_{3}\right) \delta: 0.93(3 \mathrm{H}, \mathrm{t}, J=7.5 \mathrm{~Hz}), 1.42(2 \mathrm{H}$, quint, $J=$ $7.5 \mathrm{~Hz}), 1.69(3 \mathrm{H}, \mathrm{d}, J=1.5 \mathrm{~Hz}), 1.78(2 \mathrm{H}, \mathrm{br} \mathrm{s}), 2.60-2.75(1 \mathrm{H}, \mathrm{m}), 3.44-3.62(2 \mathrm{H}, \mathrm{m}), 3.72(1 \mathrm{H}, \mathrm{dt}, J=3.0,7.5 \mathrm{~Hz})$, $3.81(3 \mathrm{H}, \mathrm{s}), 4.03(2 \mathrm{H}, \mathrm{d}, J=1.0 \mathrm{~Hz}), 4.50(1 \mathrm{H}, \mathrm{dq}, J=10.5,1.5 \mathrm{~Hz}), 6.86(2 \mathrm{H}, \mathrm{d}, J=9.0 \mathrm{~Hz}), 7.24(2 \mathrm{H}, \mathrm{d}, J=9.0 \mathrm{~Hz})$. MS $m / z$ (relative intensity): $294\left(\mathrm{M}^{+}, 1.4\right), 155(2.8), 138(22), 121(100)$. Exact MS $m / z$ Calcd for $\mathrm{C}_{17} \mathrm{H}_{26} \mathrm{O}_{4}\left(\mathrm{M}^{+}\right)$: 294.18307. Found: 294.18409. Anal. Calcd for $\mathrm{C}_{17} \mathrm{H}_{26} \mathrm{O}_{4}: \mathrm{C}, 69.36 ; \mathrm{H}, 8.90$. Found: C, 69.22; H, 9.00.

5(R)-Hydroxy-4(R)-(4-methoxybenzyl)oxymethyl-2-methyl-2(E)-heptenal (2)- $\mathrm{A} \mathrm{CH}_{2} \mathrm{Cl}_{2}$ solution (2 ml) of $11(17.0 \mathrm{mg}, 0.058 \mathrm{mmol})$ was stirred with active $\mathrm{MnO}_{2}(100 \mathrm{mg}, 1.15 \mathrm{mmol})$ at room temperature for $1 \mathrm{~h}$. After removal of the precipitate by filtration, the filtrate was evaporated in vacuo and chromatographed on a silica gel column with EtOAc-hexane $(1: 3)$ to leave 2 as a colorless oil $(16.2 \mathrm{mg}, 96 \%) .{ }^{1} \mathrm{H}-\mathrm{NMR}\left(\mathrm{CDCl}_{3}\right) \delta: 0.93(3 \mathrm{H}, \mathrm{t}, J=7.0 \mathrm{~Hz}), 1.20$ $1.60(2 \mathrm{H}, \mathrm{m}), 1.75(3 \mathrm{H}, \mathrm{d}, J=1.0 \mathrm{~Hz}), 2.7(1 \mathrm{H}, \mathrm{br} \mathrm{s}), 2.89(1 \mathrm{H}, \mathrm{ddt}, J=10.0,2.0,5.0 \mathrm{~Hz}), 3.64(2 \mathrm{H}, \mathrm{d}, J=5.0 \mathrm{~Hz}), 3.80$ $(3 \mathrm{H}, \mathrm{s}), 3.80-4.00(1 \mathrm{H}, \mathrm{m}), 4.45(2 \mathrm{H}, \mathrm{s}), 6.67(1 \mathrm{H}, \mathrm{dd}, J=10.0,1.0 \mathrm{~Hz}), 6.86(2 \mathrm{H}, \mathrm{d}, J=8.5 \mathrm{~Hz}), 7.21(2 \mathrm{H}, \mathrm{d}, J=$ $8.5 \mathrm{~Hz}), 9.41(1 \mathrm{H}, \mathrm{s})$. IR $v_{\max }^{\text {neat }} \mathrm{cm}^{-1}: 3430,1680$.

$5(S)$ - $\{5(R)$-[2-(3,4-Dimethoxybenzyloxy)ethyl]-2(S)-isopropyloxy-3(R)-methyl-6(S)-tetrahydropyranyl $\}-4(R)$ hydroxy-1-hexene (13) A solution of $12(144 \mathrm{mg}, 0.48 \mathrm{mmol})$ in THF $(2 \mathrm{ml})$ was added dropwise to a stirred suspension of $\mathrm{NaH}(100 \mathrm{mg}, 2.5 \mathrm{mmol})$ in dimethyl sulfoxide (DMSO) ( $2 \mathrm{ml}$ ) at room temperature. After $1 \mathrm{~h}, \mathrm{DMPM}$ chloride $(93.3 \mathrm{mg}, 0.50 \mathrm{mmol})$ was added, and stirring was continued for $10 \mathrm{~h}$. Then $\mathrm{Et}_{3} \mathrm{~N}(1 \mathrm{ml})$ was added, and after $30 \mathrm{~min}$, the reaction mixture was poured into saturated $\mathrm{NH}_{4} \mathrm{Cl}$ solution and extracted with $\mathrm{CH}_{2} \mathrm{Cl}_{2}$. The extract was washed with brine, dried over anhydrous $\mathrm{Na}_{2} \mathrm{SO}_{4}$, and evaporated in vacuo to leave an oil, which was chromatographed on a silica gel column with EtOAc-hexane $(1: 5)$ to give $\mathbf{1 3}$ as a colorless oil $\left(151.2 \mathrm{mg}, 70^{\circ}\right) .{ }^{1} \mathrm{H}$ $\operatorname{NMR}\left(\mathrm{CDCl}_{3}\right) \delta: 0.81(3 \mathrm{H}, \mathrm{d}, J=7 \mathrm{~Hz}), 0.91(3 \mathrm{H}, \mathrm{d}, J=7 \mathrm{~Hz}), 1.09(3 \mathrm{H}, \mathrm{d}, J=6 \mathrm{~Hz}), 1.22(3 \mathrm{H}, \mathrm{d}, J=6 \mathrm{~Hz}) .1 .15-$ $1.35(1 \mathrm{H}, \mathrm{m}), 1.47(1 \mathrm{H}, \mathrm{dt}, J=12.5,4 \mathrm{~Hz}), 1.62(1 \mathrm{H}, \mathrm{br} \mathrm{s}), 1.57-1.90(4 \mathrm{H}, \mathrm{m}), 2.14(1 \mathrm{H}, \mathrm{dt}, J=13.5,7 \mathrm{~Hz}), 2.34(1 \mathrm{H}$, $\mathrm{dt}, J=13.5,7 \mathrm{~Hz}), 3.40-3.55(2 \mathrm{H}, \mathrm{m}), 3.73(1 \mathrm{H}, \mathrm{dd}, J=2,10.5 \mathrm{~Hz}), 3.73(1 \mathrm{H}, \mathrm{d}, J=8 \mathrm{~Hz}), 3.87(1 \mathrm{H}$, sept, $J=6 \mathrm{~Hz})$, $3.88(3 \mathrm{H}, \mathrm{s}), 3.89(3 \mathrm{H}, \mathrm{s}), 4.40(1 \mathrm{H}, \mathrm{d}, J=12 \mathrm{~Hz}), 4.46(1 \mathrm{H}, \mathrm{d}, J=12 \mathrm{~Hz}), 4.67(1 \mathrm{H}, \mathrm{d}, J=3 \mathrm{~Hz}), 5.03(1 \mathrm{H}, \mathrm{d}, J=$ $10 \mathrm{~Hz}), 5.08(1 \mathrm{H}, \mathrm{d}, J=17 \mathrm{~Hz}), 5.79(1 \mathrm{H}, \mathrm{ddt}, J=10,17,7 \mathrm{~Hz}), 6.80-6.90(3 \mathrm{H}, \mathrm{m}) . \mathrm{MS} m / z$ (relative intensity): 450 $\left(\mathrm{M}^{+}, 1.0\right), 390(3.1), 319$ (1.5), 239 (2.2), 222 (19), 151 (100). Exact MS $m / z$ Calcd for $\mathrm{C}_{26} \mathrm{H}_{42} \mathrm{O}_{6}\left(\mathrm{M}^{+}\right): 450.29809$. Found: $450.29840 .[\alpha]_{\mathrm{D}}^{16}+119\left(c=0.9, \mathrm{CHCl}_{3}\right)$.

Monoacetate (colorless oil). ${ }^{1} \mathrm{H}-\mathrm{NMR}\left(\mathrm{CDCl}_{3}\right) \delta: 0.80(3 \mathrm{H}, \mathrm{d}, J=6.5 \mathrm{~Hz}), 0.88(3 \mathrm{H}, \mathrm{d}, J=7.0 \mathrm{~Hz}), 1.08(3 \mathrm{H}, \mathrm{d}$, $J=6.5 \mathrm{~Hz}), 1.19(3 \mathrm{H}, \mathrm{d}, J=6.5 \mathrm{~Hz}), 1.20-2.10(7 \mathrm{H}, \mathrm{m}), 2.03(3 \mathrm{H}, \mathrm{s}), 2.27(1 \mathrm{H}, \mathrm{dt}, J=15.5,7.5 \mathrm{~Hz}), 2.45-2.64(1 \mathrm{H}$, $\mathrm{m}), 3.46(2 \mathrm{H}, \mathrm{t}, J=6.5 \mathrm{~Hz}), 3.62(1 \mathrm{H}, \mathrm{dd}, J=5.0,1.0 \mathrm{~Hz}), 3.80-4.05(1 \mathrm{H}, \mathrm{m}), 3.88(3 \mathrm{H}, \mathrm{s}), 3.89(3 \mathrm{H}, \mathrm{s}), 4.39(1 \mathrm{H}, \mathrm{d}$, $J=11.0 \mathrm{~Hz}), 4.45(1 \mathrm{H}, \mathrm{d}, J=11.0 \mathrm{~Hz}), 4.62(1 \mathrm{H}, \mathrm{d}, J=3.0 \mathrm{~Hz}), 4.95-5.10(3 \mathrm{H}, \mathrm{m}), 5.63-5.80(1 \mathrm{H}, \mathrm{m}), 6.83-6.90$ $(3 \mathrm{H}, \mathrm{m})$.

$(4 R, 5 S, 6 S, 7 R, 9 R)-7-[2-(3,4-D i m e t h o x y b e n z y l o x y)$ ethyl]-5,9-dimethyl-4,6,10-trihydroxy-1-decene (14)-A solution of $13(1.97 \mathrm{~g}, 4.37 \mathrm{mmol})$ in THF $(50 \mathrm{ml})$ and $1 \mathrm{~N} \mathrm{HCl}(15 \mathrm{ml})$ was heated at $50^{\circ} \mathrm{C}$ for $2 \mathrm{~h}$. After neutralization with $\mathrm{NaHCO}_{3}$, the reaction mixture was evaporated in vacuo and extracted with $\mathrm{CH}_{2} \mathrm{Cl}_{2}$. The extract was dried over anhydrous $\mathrm{Na}_{2} \mathrm{SO}_{4}$ and evaporated in vacuo to leave an oil, which was chromatographed on a silica gel column with EtOAc-hexane $(1: 1)$ to give the lactol as a colorless oil $(1.70 \mathrm{~g}, 95 \%)$.

A solution of $\mathrm{NaBH}_{4}(1.5 \mathrm{~g}, 40 \mathrm{mmol})$ in $\mathrm{EtOH}(100 \mathrm{ml})$ was added dropwise to a stirred cold EtOH solution $(200 \mathrm{ml})$ of $\mathrm{CaCl}_{2}(3.0 \mathrm{~g}, 27 \mathrm{mmol})$ at $-10 \mathrm{C}$. After $30 \mathrm{~min}$, the above lactol $(1.70 \mathrm{~g}, 4.16 \mathrm{mmol})$ in EtOH $(50 \mathrm{ml})$ was added, and the stirred reaction mixture was allowed to warm to room temperature. After $1.5 \mathrm{~h}, 1 \mathrm{~N} \mathrm{HCl}$ was added to decompose the reducing agent, and the mixture was neutralized with $\mathrm{NaHCO}_{3}$. After removal of precipitated inorganic salts by filtration, the filtrate was evaporated in vacuo and extracted with $\mathrm{CH}_{2} \mathrm{Cl}_{2}$. The extract was dried over $\mathrm{Na}_{2} \mathrm{SO}_{4}$ and evaporated in vacuo to leave an oil, which was dissolved in $\mathrm{MeOH}$ containing $\mathrm{AcOH}(0.5 \mathrm{ml})$ to decompose borates. After evaporation of the solvent in vacuo, the acidic residue was neutralized with $\mathrm{NaHCO}_{3}$ and then extracted with $\mathrm{CH}_{2} \mathrm{Cl}_{2}$. The extract was dried and evaporated in vacuo to leave an oil, which was chromatographed on a silica gel column. Elution with EtOAc and then $\mathrm{CH}_{2} \mathrm{Cl}_{2}-\mathrm{MeOH}(10: 1)$ gave 14 as a colorless oil $(1.7 \mathrm{~g}, 100 \%) .{ }^{1} \mathrm{H}-\mathrm{NMR}\left(\mathrm{CDCl}_{3}\right) \delta: 0.89(6 \mathrm{H}, \mathrm{d}, J=7.0 \mathrm{~Hz}), 1.10-1.90(7 \mathrm{H}, \mathrm{m}), 2.05-2.42(2 \mathrm{H}, \mathrm{m}), 3.20-3.95$ $(9 \mathrm{H}, \mathrm{m}), 3.88(3 \mathrm{H}, \mathrm{s}), 3.89(3 \mathrm{H}, \mathrm{s}), 4.42(2 \mathrm{H}, \mathrm{s}), 5.03-5.19(2 \mathrm{H}, \mathrm{m}), 5.29-5.90(1 \mathrm{H}, \mathrm{m}), 6.84-6.90(3 \mathrm{H}, \mathrm{m})$. $[\alpha]_{\mathrm{D}}^{16}+2.3^{\circ}\left(c=1.6, \mathrm{CHCl}_{3}\right)$. MS $m / z$ (relative intensity): $410\left(\mathrm{M}^{+}, 1.5\right), 392(1), 310(1.5), 292(1.5), 282(2), 151(100)$. Exact MS $m / z$ Calcd for $\mathrm{C}_{23} \mathrm{H}_{38} \mathrm{O}_{6}\left(\mathrm{M}^{+}\right): 410.26679$. Found: 410.26802 .

$(2 R, 4 R, 5 S, 6 S, 7 R)$-4-[2-(3,4-Dimethoxybenzyloxy)ethyl]-2,6-dimethyl-5,7-isopropylidenedioxy-9-decenal (15) -A benzene solution $(50 \mathrm{ml})$ of $\mathbf{1 4}(1.70 \mathrm{~g}, 4.14 \mathrm{mmol}), 2,2$-dimethoxypropane $(5 \mathrm{ml})$ and CSA $(20 \mathrm{mg})$ was stirred at room temperature for $3 \mathrm{~h}$. The reaction mixture was washed with aqueous $\mathrm{NaHCO}_{3}$, dried over $\mathrm{Na}_{2} \mathrm{SO}_{4}$, and evaporated in vacuo to leave an oil, which was chromatographed on a silica gel column with EtOAc-hexane $(1: 3)$ to give an oil. The oil was dissolved in $\mathrm{CH}_{2} \mathrm{Cl}_{2}$ and the solution was shaken with $1 \mathrm{~N} \mathrm{HCl}$. Evaporation of the solvent gave $(4 R, 5 S, 6 S, 7 R, 9 R)$-7-[2-(3,4-dimethoxybenzyloxy)ethyl]-5,9-dimethyl-4,6-isopropylidenedioxy-1-decen-10-ol as a colorless oil $(1.56 \mathrm{~g}, 84 \%) .{ }^{1} \mathrm{H}-\mathrm{NMR}\left(\mathrm{CDCl}_{3}\right) \delta: 0.84(3 \mathrm{H}, \mathrm{d}, J=6.5 \mathrm{~Hz}), 0.85(3 \mathrm{H}, \mathrm{d}, J=6.5 \mathrm{~Hz}), 1.05-2.00(8 \mathrm{H}$, $\mathrm{m}), 1.41(3 \mathrm{H}, \mathrm{s}), 1.42(3 \mathrm{H}, \mathrm{s}), 2.01-2.42(2 \mathrm{H}, \mathrm{m}), 3.20-3.95(6 \mathrm{H}, \mathrm{m}), 3.88(3 \mathrm{H}, \mathrm{s}), 3.89(3 \mathrm{H}, \mathrm{s}), 4.43(2 \mathrm{H}, \mathrm{s}), 5.05(1 \mathrm{H}$, brd, $J=10.0 \mathrm{~Hz}), 5.08(1 \mathrm{H}, \mathrm{brd}, J=17.0 \mathrm{~Hz}), 5.53-5.79(1 \mathrm{H}, \mathrm{m}), 6.84-6.88(3 \mathrm{H}, \mathrm{m}) . \mathrm{MS} m / z$ (relative intensity): $450\left(\mathrm{M}^{+}, 1.5\right), 398$ (1.5), 310 (3.5), 204 (3.1), 151 (100). Exact MS $m / z$ Calcd for $\mathrm{C}_{26} \mathrm{H}_{42} \mathrm{O}_{6}\left(\mathrm{M}^{+}\right): 450.29809$. Found: 450.29810. $[\alpha]_{\mathrm{D}}^{15}+16^{\circ}\left(c=2.0, \mathrm{CHCl}_{3}\right)$. 
A solution of DMSO $(95 \mathrm{mg}, 1.2 \mathrm{mmol})$ in $\mathrm{CH}_{2} \mathrm{Cl}_{2}(1 \mathrm{ml})$ was added to a cold stirred $\mathrm{CH}_{2} \mathrm{Cl}_{2}$ solution of $(\mathrm{COCl})_{2}$ ( $77 \mathrm{mg}, 0.6 \mathrm{mmol})$ at $-80 \mathrm{C}$, and then the above alcohol $(182 \mathrm{mg}, 0.40 \mathrm{mmol})$ in $\mathrm{CH}_{2} \mathrm{Cl}_{2}$ was similarly added. After $1 \mathrm{~h}, \mathrm{Et}_{3} \mathrm{~N}(134 \mathrm{mg}, 1.3 \mathrm{mmol})$ was added, then the reaction mixture was allowed to warm to room temperature, washed with $\mathrm{H}_{2} \mathrm{O}, 0.5 \mathrm{~N} \mathrm{HCl}$, brine and aqueous $\mathrm{NaHCO}_{3}$, dried over $\mathrm{Na}_{2} \mathrm{SO}_{4}$, and evaporated to leave 15 as an oil $(172 \mathrm{mg}, 94 \%) .{ }^{1} \mathrm{H}-\mathrm{NMR}\left(\mathrm{CDCl}_{3}\right) \delta: 0.83(3 \mathrm{H}, \mathrm{d}, J=7.0 \mathrm{~Hz}), 1.04(3 \mathrm{H}, \mathrm{d}, J=7.0 \mathrm{~Hz}), 1.10-2.70(9 \mathrm{H}, \mathrm{m}), 1.35(3 \mathrm{H}$, s), $1.38(3 \mathrm{H}, \mathrm{s}), 3.4 \mathrm{l}-3.95(4 \mathrm{H}, \mathrm{m}) .3 .88(3 \mathrm{H}, \mathrm{s}), 3.89(3 \mathrm{H}, \mathrm{s}), 4.42(2 \mathrm{H}, \mathrm{s}), 5.04(1 \mathrm{H}, \mathrm{br} \mathrm{d}, J=10.0 \mathrm{~Hz}), 5.08(1 \mathrm{H}, \mathrm{br}$, $J=17.0 \mathrm{~Hz}), 5.53-5.87(1 \mathrm{H}, \mathrm{m}), 6.84-6.88(3 \mathrm{H}, \mathrm{m}), 9.55(1 \mathrm{H}, \mathrm{d}, J=2.0 \mathrm{~Hz})$. IR $v_{\max }^{\text {neat }} \mathrm{cm}^{-1}: 1720$.

Dimethyl $\quad(3 R, 5 R, 6 S, 7 S, 8 R)-5$-[2-(3,4-Dimethoxybenzyloxy)ethyl]-3,7-dimethyl-6,8-isopropylidenedioxy-2-oxo10-undecenylphosphonate (16) - A $1.6 \mathrm{M} \mathrm{BuLi}$ hexane solution $(440 \mu \mathrm{l})$ was added to a stirred THF solution (6 ml) of dimethyl methylphosphonate $(120 \mu \mathrm{l}, 1.0 \mathrm{mmol})$ at $-70{ }^{\circ} \mathrm{C}$ under argon. After $30 \mathrm{~min}, 15(172 \mathrm{mg}, 0.40 \mathrm{mmol})$ in THF was added dropwise, then the reaction mixture was allowed to warm to $-30 \mathrm{C}$, quenched by the addition of saturated $\mathrm{NH}_{4} \mathrm{Cl}$, and extracted with ether. The extract was washed with brine, dried over anhydrous $\mathrm{Na}_{2} \mathrm{SO}_{4}$, and evaporated to leave the hydroxyphosphonate as a colorless oil $(215 \mathrm{mg}, 98 \%)$, which was dissolved in DMF $(6.5 \mathrm{ml})$. PDC $(500 \mathrm{mg})$ was added to the resulting solution, and the mixture was stirred for $1 \mathrm{~d}$. Further PDC (500 mg) was added and stirring was continued for $5 \mathrm{~h}$. The reaction mixture was poured into $\mathrm{H}_{2} \mathrm{O}$ and extracted with ether. The extract was washed with brine, dried over anhydrous $\mathrm{Na}_{2} \mathrm{SO}_{4}$, and evaporated to leave an oil, which was chromatographed on a silica gel column with EtOAc-hexane $(2: 1)$ to give 16 as a colorless oil $(146 \mathrm{mg}, 67 \%) .{ }^{1} \mathrm{H}-$ $\operatorname{NMR}\left(\mathrm{CDCl}_{3}\right) \delta: 0.84(3 \mathrm{H}, \mathrm{d}, J=7.0 \mathrm{~Hz}), 1.06(3 \mathrm{H}, \mathrm{d}, J=7.0 \mathrm{~Hz}), 1.10-1.90(6 \mathrm{H}, \mathrm{m}), 1.40(3 \mathrm{H}, \mathrm{s}), 1.41(3 \mathrm{H}, \mathrm{s}), 2.11$ $(1 \mathrm{H}, \mathrm{dt}, J=15.0,7.0 \mathrm{~Hz}), 2.29(1 \mathrm{H}, \mathrm{dt}, J=15.0,7.0 \mathrm{~Hz}), 2.84-3.04(1 \mathrm{H}, \mathrm{m}), 3.15(2 \mathrm{H}, \mathrm{d}, J=22.0 \mathrm{~Hz}), 3.45-3.90$ $(4 \mathrm{H}, \mathrm{m}), 3.74(3 \mathrm{H}, \mathrm{d}, J=1.0 \mathrm{~Hz}), 3.80(3 \mathrm{H}, \mathrm{d}, J=10.0 \mathrm{~Hz}), 3.88(3 \mathrm{H}, \mathrm{m}), 3.89(3 \mathrm{H}, \mathrm{s}), 4.41(2 \mathrm{H}, \mathrm{s}), 5.04(1 \mathrm{H}, \mathrm{d}, J=$ $9.0 \mathrm{~Hz}), 5.10(1 \mathrm{H}, \mathrm{d}, J=15.0 \mathrm{~Hz}), 5.50-5.90(1 \mathrm{H}, \mathrm{m}), 6.82-6.89(3 \mathrm{H}, \mathrm{m})$. IR $v_{\max }^{\text {nea }} \mathrm{cm}^{-1}: 1715$. MS $m / z$ (relative intensity): $570\left(\mathrm{M}^{+}, 0.7\right), 442(0.5), 232(12), 193(12), 180(23), 151(100)$. Exact $\mathrm{MS} m / z$ Calcd for $\mathrm{C}_{29} \mathrm{H}_{47} \mathrm{O}_{9} \mathrm{P}\left(\mathrm{M}^{+}\right)$: 570.29571. Found: 570.29603.

$(3 R, 4 S, 5 S, 6 R, 8 R)-6$-[2-(3,4-Dimethoxybenzyloxy)ethyl]-10-(dimethoxyphosphono)-4,8-dimethyl-3,5-isopropylidenedioxy-9-oxodecanoic Acid (3)-A solution of $16(95 \mathrm{mg}, 0.16 \mathrm{mmol})$ in acetone, $10 \%$ aqueous $\mathrm{NaHCO}_{3}$ $(0.2 \mathrm{ml})$ and a solution of $\mathrm{KMnO}_{4}(5 \mathrm{mg}, 0.03 \mathrm{mmol})$ in $\mathrm{H}_{2} \mathrm{O}(0.2 \mathrm{ml})$ were added successively to a stirred aqueous solution $(5 \mathrm{ml})$ of $\mathrm{NaIO}_{4}(340 \mathrm{mg}, 1.6 \mathrm{mmol})$ at room temperature. After $5 \mathrm{~h}$, the reaction mixture was filtered, then the filtrate was mixed with saturated $\mathrm{NH}_{4} \mathrm{Cl}$ solution $(10 \mathrm{ml})$, and the mixture was extracted with $\mathrm{CH}_{2} \mathrm{Cl}_{2}$. The extract was washed with aqueous $\mathrm{NH}_{4} \mathrm{Cl}$ and brine, dried over anhydrous $\mathrm{Na}_{2} \mathrm{SO}_{4}$, and evaporated in vacuo to leave 3 as a colorless viscous oil $(82 \mathrm{mg}, 83 \%) .{ }^{1} \mathrm{H}-\mathrm{NMR}\left(\mathrm{CDCl}_{3}\right) \delta: 0.86(3 \mathrm{H}, \mathrm{d}, J=7.0 \mathrm{~Hz}), 1.06(3 \mathrm{H}, \mathrm{d}, J=7.0 \mathrm{~Hz}), 1.10-2.00$ $(6 \mathrm{H}, \mathrm{m}), 1.39(3 \mathrm{H}, \mathrm{s}), 1.44(3 \mathrm{H}, \mathrm{s}), 2.37(1 \mathrm{H}, \mathrm{dd}, J=16.0,5.0 \mathrm{~Hz}), 2.58(1 \mathrm{H}, \mathrm{dd}, J=16.0,8.0 \mathrm{~Hz}), 2.80-3.20(1 \mathrm{H}, \mathrm{m})$, $3.14(2 \mathrm{H}, \mathrm{d}, J=22.0 \mathrm{~Hz}), 3.47(2 \mathrm{H}, \mathrm{t}, J=6.0 \mathrm{~Hz}), 3.72(3 \mathrm{H}, \mathrm{d}, J=1.0 \mathrm{~Hz}), 3.83(3 \mathrm{H}, \mathrm{d}, J=1.0 \mathrm{~Hz}), 3.85-4.00(1 \mathrm{H}, \mathrm{m})$, $3.88(3 \mathrm{H}, \mathrm{s}), 3.89(3 \mathrm{H}, \mathrm{s}), 4.20-4.40(1 \mathrm{H}, \mathrm{m}), 4.41(2 \mathrm{H}, \mathrm{s}), 6.85(3 \mathrm{H}, \mathrm{s}) . I_{\mathrm{R}} v_{\max }^{\text {neat }} \mathrm{cm}^{-1}: 1720,1710 .[\alpha]_{\mathrm{D}}^{26.5}-5.6^{\circ}(c=$ $\left.1.4, \mathrm{CHCl}_{3}\right)$.

$1(R)$-Ethyl-5-formyl-2(R)-(4-methoxybenzyl)oxymethyl-3(Z)-pentenyl $\quad(3 R, 4 S, 5 S, 6 R, 8 R)-6$-[2-(3,4-Dimethoxybenzyl)oxyethyl]-10-(dimethoxyphosphono)-4,8-dimethyl-3,5-isopropylidenedioxy-9-oxodecanoate (17)-2,4,6-Trichlorobenzoyl chloride $(10 \mu \mathrm{l}, 0.06 \mathrm{mmol})$ was added dropwise to a stirred THF solution $(0.5 \mathrm{ml})$ of 3 (35 mg, $0.059 \mathrm{mmol})$ and $\mathrm{Et}_{3} \mathrm{~N}(10 \mu \mathrm{l}, 0.07 \mathrm{mmol})$ at room temperature under argon. After $20 \mathrm{~min}$, the precipitates were filtered off, and the filtrate was evaporated in vacuo. The residue was dissolved in benzene ( $1 \mathrm{ml})$ and added dropwise to a stirred benzene solution $(0.5 \mathrm{ml})$ of $2(25 \mathrm{mg}, 0.086 \mathrm{mmol})$ and DMAP $(9 \mathrm{mg}, 0.07 \mathrm{mmol})$ at room temperature. After $1 \mathrm{~h}$, the reaction mixture was washed with brine and $10 \% \mathrm{NaHCO}_{3}$ solution, dried over anhydrous $\mathrm{Na}_{2} \mathrm{SO}_{4}$, and evaporated to leave an oil, which was subjected to silica gel preparative thin layer chromatography (TLC) with EtOAc-hexane (2:1) to give 17 as a colorless oil $(34.1 \mathrm{mg}, 66 \%) .{ }^{1} \mathrm{H}-\mathrm{NMR}\left(\mathrm{CDCl}_{3}\right) \delta: 0.84(3 \mathrm{H}, \mathrm{d}, J=7.0 \mathrm{~Hz}), 0.86$ $(3 \mathrm{H}, \mathrm{t}, J=7.5 \mathrm{~Hz}), 1.06(3 \mathrm{H}, \mathrm{d}, J=7.0 \mathrm{~Hz}), 1.10-2.00(8 \mathrm{H}, \mathrm{m}), 1.36(3 \mathrm{H}, \mathrm{s}), 1.41(3 \mathrm{H}, \mathrm{s}), 1.78(3 \mathrm{H}, \mathrm{s}), 2.21(1 \mathrm{H}, \mathrm{dd}$, $J=15.0,4.0 \mathrm{~Hz}), 2.51(1 \mathrm{H}, \mathrm{dd}, J=15.0,9.0 \mathrm{~Hz}), 2.85-3.25(2 \mathrm{H}, \mathrm{m}), 3.13(2 \mathrm{H}, \mathrm{d}, J=22.0 \mathrm{~Hz}), 3.38-3.60(4 \mathrm{H}, \mathrm{m})$, $3.63-4.05(2 \mathrm{H}, \mathrm{m}), 3.74(3 \mathrm{H}, \mathrm{d}, J=1.0 \mathrm{~Hz}), 3.80(6 \mathrm{H}, \mathrm{s}), 3.87(3 \mathrm{H}, \mathrm{s}), 3.88(3 \mathrm{H}, \mathrm{s}), 4.32-4.45(4 \mathrm{H}, \mathrm{m}), 5.21(1 \mathrm{H}, \mathrm{m})$, $6.45(1 \mathrm{H}, \mathrm{d}, J=10.0 \mathrm{~Hz}), 6.80-6.95(5 \mathrm{H}, \mathrm{m}), 7.20(2 \mathrm{H}, \mathrm{d}, J=9.0 \mathrm{~Hz}), 9.44(1 \mathrm{H}, \mathrm{s}) . \mathrm{MS} m / z$ (relative intensity): 741 $\left(\mathrm{M}^{+}-121,0.9\right), 683(1.5), 363(2.0), 345(3.3), 263(10), 232$ (15), $180(22), 151$ (100). Exact MS $m / z$ Calcd for $\mathrm{C}_{34} \mathrm{H}_{52} \mathrm{O}_{12} \mathrm{P}\left(\mathrm{M}^{+}-179\right): 683.31956$. Found: 683.32219. IR $v_{\max }^{\text {neat }} \mathrm{cm}^{-1}: 1735,1710,1690 .[\alpha]_{\mathrm{D}}^{26}+2.8^{\circ} \cdot\left(c=1.3, \mathrm{CHCl}_{3}\right)$.

6"-Dihydro-6"-O-(3,4-dimethoxybenzyl)-3,5-O-isopropylidene-14'-O-(4-methoxybenzyl)tylonolide (18) - A toluene solution $(5 \mathrm{ml})$ of $17(34.1 \mathrm{mg}, 0.039 \mathrm{mmol})$ was added very slowly to a stirred suspension of $\mathrm{K}_{2} \mathrm{CO}_{3}(33 \mathrm{mg}$, $0.24 \mathrm{mmol})$ in toluene $(50 \mathrm{ml})$ in the presence of 18 -crown- $6(125 \mathrm{mg}, 0.47 \mathrm{mmol})$ at $100{ }^{\circ} \mathrm{C}$ during $\mathrm{l} \mathrm{h}$, and stirring was continued at the same temperature for $20 \mathrm{~h}$. After cooling to room temperature, the reaction mixture was mixed with saturated $\mathrm{NH}_{4} \mathrm{Cl}$ solution and extracted with ether. The extract was washed with saturated $\mathrm{KCl}$ solution several times, dried over $\mathrm{Na}_{2} \mathrm{SO}_{4}$, and evaporated to leave an oil, which was purified by silica gel preparative TLC with EtOAc-hexane $(1: 2)$ to give 18 as a colorless oil $(17.2 \mathrm{mg}, 59 \%) .{ }^{1} \mathrm{H}-\mathrm{NMR}\left(\mathrm{CDCl}_{3}\right) \delta: 0.83(3 \mathrm{H}, \mathrm{d}, J=6.5 \mathrm{~Hz}), 0.88$ $(3 \mathrm{H}, \mathrm{t}, J=7.0 \mathrm{~Hz}), 1.10-2.20(8 \mathrm{H}, \mathrm{m}), 1.13(3 \mathrm{H}, \mathrm{d}, J=6.5 \mathrm{~Hz}), 1.38(3 \mathrm{H}, \mathrm{s}), 1.43(3 \mathrm{H}, \mathrm{s}), 1.82(3 \mathrm{H}, \mathrm{s}), 2.34(2 \mathrm{H}, \mathrm{t}, J=$ $5.0 \mathrm{~Hz}), 2.80-3.10(3 \mathrm{H}, \mathrm{m}), 3.40(2 \mathrm{H}, \mathrm{t}, J=6.5 \mathrm{~Hz}), 3.46(2 \mathrm{H}, \mathrm{d}, J=5.0 \mathrm{~Hz}), 3.81(3 \mathrm{H}, \mathrm{s}), 3.90(3 \mathrm{H}, \mathrm{s}), 3.90-4.20$ $(2 \mathrm{H}, \mathrm{m}), 4.35-4.50(4 \mathrm{H}, \mathrm{m}), 4.99(1 \mathrm{H}, \mathrm{dt}, J=4.0,7.5 \mathrm{~Hz}), 5.81(1 \mathrm{H}, \mathrm{d}, J=11.0 \mathrm{~Hz}), 6.17(1 \mathrm{H}, \mathrm{d}, J=16.0 \mathrm{~Hz}), 6.80-$ $6.95(5 \mathrm{H}, \mathrm{m}), 7.17(1 \mathrm{H}, \mathrm{d}, J=16.0 \mathrm{~Hz}), 7.20(2 \mathrm{H}, \mathrm{d}, J=9.0 \mathrm{~Hz}) . \mathrm{MS} m / z$ (relative intensity): $736\left(\mathrm{M}^{+}, 0.55\right), 600(0.5)$, 
585 (0.9), 512 (0.95), 376 (3.0), 165 (13), 151 (87), 121 (100). Exact MS $m / z$ Calcd for $\mathrm{C}_{34} \mathrm{H}_{49} \mathrm{O}_{8}\left(\mathrm{M}^{+}-151\right)$ : 585.34268. Found: 585.33994. FD-MS $m / z$ (relative intensity): $736\left(\mathrm{M}^{+}, 100\right)$.

6"'-Dihydro-3,5-O-isopropylidene-14'-O-(4-methoxybenzyl)tylonolide (19) - A $4 \%$ benzene solution on DDQ ( $77 \mu \mathrm{l}, 3.1 \mathrm{mg}, 0.014 \mathrm{mmol})$ was added to a stirred ice-cold solution of $18(10.0 \mathrm{mg}, 0.0136 \mathrm{mmol})$ in $\mathrm{CH}_{2} \mathrm{Cl}_{2}(1.0 \mathrm{ml})$ and $\mathrm{H}_{2} \mathrm{O}(0.05 \mathrm{ml})$, and stirring was continued for $3 \mathrm{~h}$ at $0-5{ }^{\circ} \mathrm{C}$. The reaction mixture was poured into aqueous $\mathrm{NaHCO}_{3}$, then the organic layer was washed with aqueous $\mathrm{NaHCO}_{3}$, dried over anhydrous $\mathrm{Na}_{2} \mathrm{SO}_{4}$, and evaporated in vacuo to leave an oil, which was purified by silica gel TLC with EtOAc-hexane (1:1) to give 19 as a colorless oil $(6.0 \mathrm{mg}, 75 \%) .{ }^{1} \mathrm{H}-\mathrm{NMR}\left(\mathrm{CDCl}_{3}\right) \delta: 0.86(3 \mathrm{H}, \mathrm{d}, J=7.0 \mathrm{~Hz}), 0.89(3 \mathrm{H}, \mathrm{t}, J=7.0 \mathrm{~Hz}), 1.10-2.00(8 \mathrm{H}, \mathrm{m}), 1.18(3 \mathrm{H}, \mathrm{d}$, $J=6.5 \mathrm{~Hz}), 1.40(3 \mathrm{H}, \mathrm{s}), 1.45(3 \mathrm{H}, \mathrm{s}), 1.82(3 \mathrm{H}, \mathrm{s}), 2.34(2 \mathrm{H}, \mathrm{t}, J=6.5 \mathrm{~Hz}), 2.80-3.03(3 \mathrm{H}, \mathrm{m}), 3.40-3.80(2 \mathrm{H}, \mathrm{m})$, $3.46(2 \mathrm{H}, \mathrm{d}, J=5.0 \mathrm{~Hz}), 3.81(3 \mathrm{H}, \mathrm{s}), 3.93(1 \mathrm{H}, \mathrm{br} \mathrm{s}), 4.05(1 \mathrm{H}, \mathrm{t}, J=3 \mathrm{~Hz}), 4.20(1 \mathrm{H}, \mathrm{m}), 4.41(2 \mathrm{H}, \mathrm{ABq}, J=10.0 \mathrm{~Hz})$, $5.01(1 \mathrm{H}, \mathrm{dt}, J=4.0,7.5 \mathrm{~Hz}), 5.83(1 \mathrm{H}, \mathrm{d}, J=10.5 \mathrm{~Hz}), 6.21(1 \mathrm{H}, \mathrm{d}, J=16.0 \mathrm{~Hz}), 6.87(2 \mathrm{H}, \mathrm{d}, J=9.0 \mathrm{~Hz}), 7.15(1 \mathrm{H}, \mathrm{d}$, $J=16.0 \mathrm{~Hz}$ ), $7.23(2 \mathrm{H}, \mathrm{d}, J=9.0 \mathrm{~Hz})$. IR $v_{\max }^{\mathrm{CHCl}_{3}} \mathrm{~cm}^{-1}: 3400,1720,1680$. MS $m / z$ (relative intensity): $586\left(\mathrm{M}^{+}, 0.7\right), 528$ (0.8), 450 (4.3), 392 (2.3), 135 (16), 121 (100). Exact MS $m / z$ Calcd for $\mathrm{C}_{34} \mathrm{H}_{50} \mathrm{O}_{8}\left(\mathrm{M}^{+}\right)$: 586.35051. Found: 586.34885.

14'-O-(4-Methoxybenzyl)tylonolide Hemiacetal (20)- $\mathrm{A} \mathrm{CH}_{2} \mathrm{Cl}_{2}$ solution $(2 \mathrm{ml})$ of $19(8.3 \mathrm{mg}, 0.014 \mathrm{mmol})$ and PDC $(30 \mathrm{mg}, 0.08 \mathrm{mmol})$ was stirred at room temperature for $6 \mathrm{~h}$. The reaction mixture was directly chromatographed on a silica gel column with ether to give 14'-O-(4-methoxybenzyl)-3,5- $O$-isopropylidenetylonolide as a colorless oil $(7.5 \mathrm{mg}, 91 \%) .{ }^{1} \mathrm{H}-\mathrm{NMR}\left(\mathrm{CDCl}_{3}\right) \delta: 0.84(3 \mathrm{H}, \mathrm{d}, J=6.5 \mathrm{~Hz}), 1.36(3 \mathrm{H}, \mathrm{s}), 1.43(3 \mathrm{H}, \mathrm{s}), 1.81(3 \mathrm{H}, \mathrm{d}, J=$ $1.0 \mathrm{~Hz}), 2.70-3.10(4 \mathrm{H}, \mathrm{m}), 3.46(2 \mathrm{H}, \mathrm{d}, J=5.0 \mathrm{~Hz}), 3.81(3 \mathrm{H}, \mathrm{s}), 3.85-4.15(2 \mathrm{H}, \mathrm{m}), 4.41(2 \mathrm{H}, \mathrm{s}), 5.04(1 \mathrm{H}, \mathrm{dt}, J=$ $4.0,7.5 \mathrm{~Hz}), 5.85(1 \mathrm{H}, \mathrm{d}, J=10.5 \mathrm{~Hz}), 6.17(1 \mathrm{H}, \mathrm{d}, J=16.0 \mathrm{~Hz}), 6.86(2 \mathrm{H}, \mathrm{d}, J=9.0 \mathrm{~Hz}), 7.17(1 \mathrm{H}, \mathrm{d}, J=16.0 \mathrm{~Hz}), 7.25$ $(2 \mathrm{H}, \mathrm{d}, J=9.0 \mathrm{~Hz}), 9.64(1 \mathrm{H}, \mathrm{t}, J=2.0 \mathrm{~Hz})$. MS $m / z$ (relative intensity): $584\left(\mathrm{M}^{+}, 0.4\right), 448(2.0), 135(17), 121(100)$. Exact MS $m / z$ Calcd for $\mathrm{C}_{34} \mathrm{H}_{48} \mathrm{O}_{8}\left(\mathrm{M}^{+}\right)$: 584.33486 . Found: 584.33424 .

This oil was dissolved in THF $(2 \mathrm{ml})$ and $0.5 \mathrm{~N} \mathrm{HCl}(0.5 \mathrm{ml})$ and stirred for $1 \mathrm{~h}$ at room temperature. The reaction mixture was neutralized with $\mathrm{NaHCO}_{3}$ and extracted with ether. The extract was washed with brine, dried over $\mathrm{Na}_{2} \mathrm{SO}_{4}$, and evaporated in vacuo to leave an oil, which was chromatographed on a silica gel column with EtOAchexane $(1: 1)$ to give 20 as a colorless oil $(5.6 \mathrm{mg}, 90 \%) .{ }^{1} \mathrm{H}-\mathrm{NMR}\left(\mathrm{CDCl}_{3}\right) \delta: 0.83(3 \mathrm{H}, \mathrm{d}, J=7.5 \mathrm{~Hz}), 0.97(3 \mathrm{H}, \mathrm{t}, J=$ $7.0 \mathrm{~Hz}), 1.10-2.25(9 \mathrm{H}, \mathrm{m}), 1.23(3 \mathrm{H}, \mathrm{d}, J=7.0 \mathrm{~Hz}), 1.80(3 \mathrm{H}, \mathrm{d}, J=1.0 \mathrm{~Hz}), 2.27-2.70(2 \mathrm{H}, \mathrm{m}), 2.75-3.12(1 \mathrm{H}, \mathrm{m})$, $3.49(2 \mathrm{H}, \mathrm{d}, J=5.0 \mathrm{~Hz}), 3.60(1 \mathrm{H}, \mathrm{d}, J=10.5 \mathrm{~Hz}), 3.77-3.90(1 \mathrm{H}, \mathrm{m}), 3.81(3 \mathrm{H}, \mathrm{s}), 4.12(1 \mathrm{H}, \mathrm{dd}, J=10.0,4.0 \mathrm{~Hz})$, $4.44(2 \mathrm{H}, \mathrm{s}), 4.93(1 \mathrm{H}, \mathrm{dt}, J=2.0,10.0 \mathrm{~Hz}), 5.35-5.60(1 \mathrm{H}, \mathrm{m}), 5.80(1 \mathrm{H}, \mathrm{d}, J=10.5 \mathrm{~Hz}), 6.33(0.8 \mathrm{H}, \mathrm{d}, J=16.0 \mathrm{~Hz})$, $6.37(0.2 \mathrm{H}, \mathrm{d}, J=16.0 \mathrm{~Hz}), 6.86(2 \mathrm{H}, \mathrm{d}, J=9.0 \mathrm{~Hz}), 7.22(2 \mathrm{H}, \mathrm{d}, J=9.0 \mathrm{~Hz}), 7.23(1 \mathrm{H}, \mathrm{d}, J=16.0 \mathrm{~Hz}) . \mathrm{MS} m / z$ (relative intensity): $526\left(\mathrm{M}^{+}, 0.5\right), 508(0.5), 405(2.8), 387(2.0), 207(2.9), 135(11), 121$ (100). Exact MS $m / z$ Calcd for $\mathrm{C}_{31} \mathrm{H}_{42} \mathrm{O}_{7}\left(\mathrm{M}^{+}\right)$: 526.29930. Found: 526.29386.

Tylonolide Hemiacetal (1)-A solution of $20(5.6 \mathrm{mg}, 0.010 \mathrm{mmol})$ and DDQ $(3.5 \mathrm{mg}, 0.015 \mathrm{mmol})$ in $\mathrm{CH}_{2} \mathrm{Cl}_{2}$ $(1 \mathrm{ml})$ and $\mathrm{H}_{2} \mathrm{O}(0.05 \mathrm{ml})$ was stirred at room temperature for $6 \mathrm{~h}$, then poured into aqueous $\mathrm{NaHCO}_{3}$ and extracted with $\mathrm{CH}_{2} \mathrm{Cl}_{2}$. The extract was dried over anhydrous $\mathrm{Na}_{2} \mathrm{SO}_{4}$, and evaporated in vacuo. The residue was subjected to silica TLC with EtOAc-hexane $(2: 1)$ to give 1 as a colorless solid $(3.0 \mathrm{mg}, 70 \%), \mathrm{mp} 103 \mathrm{C} .{ }^{1} \mathrm{H}-\mathrm{NMR}\left(\mathrm{CDCl}_{3}\right) \delta$ $(500 \mathrm{MHz}): 0.95(3 \mathrm{H}, \mathrm{t}, J=7 \mathrm{~Hz}, \mathrm{C}-17), 1.02\left(3 \mathrm{H}, \mathrm{d}, J=7 \mathrm{~Hz}, \mathrm{C}-8^{\prime}\right), 1.05(1 \mathrm{H}, \mathrm{ddd}, J=4,12,16 \mathrm{~Hz}, \mathrm{C}-7), 1.23(3 \mathrm{H}, \mathrm{d}$, $\left.J=7 \mathrm{~Hz}, \mathrm{C}-4^{\prime}\right), 1.60-1.73(3 \mathrm{H}, \mathrm{m}, \mathrm{C}-7(1 \mathrm{H}), \mathrm{C}-16(2 \mathrm{H})), 1.55(1 \mathrm{H}, \mathrm{dq}, J=7,10 \mathrm{~Hz}, \mathrm{C}-4), 1.80-1.95\left(1 \mathrm{H}, \mathrm{m}, \mathrm{C}^{\prime} 6^{\prime}\right)$, $1.80\left(3 \mathrm{H}, \mathrm{s}, \mathrm{C}-12^{\prime}\right), 1.94(1 \mathrm{H}, \mathrm{dd}, J=2,17 \mathrm{~Hz}, \mathrm{C}-2), 2.01-2.07(1 \mathrm{H}, \mathrm{m}, \mathrm{C}-6), 2.19\left(0.23 \mathrm{H}, \mathrm{dd}, J=6,13 \mathrm{~Hz}, \mathrm{C}-6^{\prime}\right), 2.27$ $\left(0.77 \mathrm{H}, \mathrm{dd}, J=6,13 \mathrm{~Hz}, \mathrm{C}-6^{\prime}\right), 2.45-2.65(1 \mathrm{H}, \mathrm{m}, \mathrm{C}-8), 2.57(1 \mathrm{H}, \mathrm{dd}, J=11,17 \mathrm{~Hz}, \mathrm{C}-2), 2.88(1 \mathrm{H}, \mathrm{ddt}, J=5,6$, $10 \mathrm{~Hz}, \mathrm{C}-14), 3.67(1 \mathrm{H}, \mathrm{dd}, J=2,11 \mathrm{~Hz}, \mathrm{C}-3), 3.68-3.77\left(2 \mathrm{H}, \mathrm{m}, \mathrm{C}-14^{\prime}\right), 3.80(0.23 \mathrm{H}, \mathrm{dd}, J=4,10 \mathrm{~Hz}, \mathrm{C}-5), 4.12$ $(0.77 \mathrm{H}, \mathrm{dd}, J=4,10 \mathrm{~Hz}, \mathrm{C}-5), 4.93(1 \mathrm{H}, \mathrm{dt}, J=2.10 \mathrm{~Hz}, \mathrm{C}-15), 5.42\left(0.23 \mathrm{H}, \mathrm{d}, J=6 \mathrm{~Hz}, \mathrm{C}-6^{\prime \prime}\right), 5.50(0.77 \mathrm{H}, \mathrm{dd}, J=4$, $\left.6 \mathrm{~Hz}, \mathrm{C}-6^{\prime \prime}\right), 5.80(1 \mathrm{H}, \mathrm{d}, J=10 \mathrm{~Hz}, \mathrm{C}-13), 6.33(0.77 \mathrm{H}, \mathrm{d}, J=15 \mathrm{~Hz}, \mathrm{C}-10), 6.37(0.23 \mathrm{H}, \mathrm{d}, J=15 \mathrm{~Hz}, \mathrm{C}-10), 7.22(1 \mathrm{H}$, $\mathrm{d}, J=15 \mathrm{~Hz}, \mathrm{C}-11)$. MS $m / z$ (relative intensity): $424\left(\mathrm{M}^{+}, 2\right), 406$ (17), 393 (12), 388 (12), 191 (39), 121 (100). Exact MS $m / z$ Calcd for $\mathrm{C}_{23} \mathrm{H}_{36} \mathrm{O}_{7}\left(\mathrm{M}^{+}\right): 424.24605$. Found: 424.24538 .

Acknowledgement We are grateful to Professor M. Yamaguchi and Dr. J. Inanaga, Kyushu University, for their kind gift of the precious reagent, 2,4,6-trichlorobenzoyl chloride.

\section{References and Notes}

1) Chiral Syntheses of Polyketide-Derived Natural Products. XV. For part XIV, see: T. Tanaka, Y. Oikawa, T. Hamada, and O. Yonemitsu, Chem. Pharm. Bull., 35, 2209 (1987).

2) A preliminary report on this work: T. Tanaka, Y. Oikawa, T. Hamada, and O. Yonemitsu, Tetrahedron Lett., 27, 3651 (1986).

3) a) K. Tatsuta, Y. Amemiya, Y. Kanemaru, and M. Kinoshita, Tetrahedron Lett., 22, 3997 (1981); b) K. C. Nicolaou, M. R. Pavia, and S. P. Seitz, J. Am. Chem. Soc., 104, 2027, 2030 (1982); c) S. Masamune, L. D.-L. Lu, T. Kaiho, and T. Toyoda, ibid., 104, 5523 (1982); d) P. A. Grieco, J. Inanaga, N.-H. Lin, and T. Yanami, ibid., 104, 5781 (1982).

4) a) Y. Oikawa, T. Tanaka, K. Horita, I. Noda, N. Nakajima, N. Kakusawa, T. Hamada, and O. Yonemitsu, Chem. Pharm. Bull., 35, 2184 (1987); b) Y. Oikawa, T. Tanaka, T. Hamada, and O. Yonemitsu, ibid., 35, 2196 
(1987); c) T. Tanaka, Y. Oikawa, N. Nakajima, T. Hamada, and O. Yonemitsu, ibid., 35, 2203 (1987).

5) Y. Oikawa, T. Yoshioka, and O. Yonemitsu, Tetrahedron Lett., 23, 885 (1982).

6) K. Horita, T. Yoshioka, T. Tanaka, Y. Oikawa, and O. Yonemitsu, Tetrahedron, 42, 3021 (1986), and references cited therein.

7) Y. Oikawa, T. Tanaka, K. Horita, T. Yoshioka, and O. Yonemitsu, Tetrahedron Lett., 25, 5393 (1984).

8) Unless otherwise noted, the numberings are based on that of tylonolide (1).

9) Y. Oikawa, T. Nishi, and O. Yonemitsu, J. Chem. Soc., Perkin Trans. 1, 1985, 19.

10) The MPM ether was synthesized by the usual MPM protection ${ }^{5,6)}$ of the corresponding alcohol, ${ }^{11)}$ which was derived from the olefin precursor by hydroboration in $83 \%$ yield. The olefin was synthesized from the ketone by usual Wittig reaction with $\mathrm{Ph}_{3} \mathrm{P}=\mathrm{CH}_{2}$, which was prepared from $\mathrm{Ph}_{3} \mathrm{PCH}_{3} \mathrm{Br}$ and $n$-BuLi. ${ }^{11}$ When the olefin was synthesized by use of the Wittig reagent prepared from $\mathrm{Ph}_{3} \mathrm{PCH}_{3} \mathrm{Br}$ with $\mathrm{MeSOCH}_{2} \mathrm{Na}$, the hydroboration of the olefin proceeded very slowly to give the alcohol in rather poor yield. ${ }^{11}$

11) A. Rosental and M. Sprinzl, Can. J. Chem., 47, 4477 (1969).

12) The numbering is based on that of D-glucose.

13) A. Rosental and M. Sprinzl, Can. J. Chem., 47, 3941 (1969).

14) L. Leval, G. Fordor, K. Ritray-Emandity, O. Fuchs, and A. Hajos, Chem. Ber., 93, 387 (1960).

15) Other reducing agents such as $\mathrm{LiAlH}_{4}$ and $\mathrm{NaBH}_{4}$ gave only unsatisfactory results due to poor reproducibility and incomplete reaction.

16) O. Isler, H. Gutmann, M. Montavon, R. Ruegg, G. Ryser, and P. Zeller, Helv. Chim. Acta, 40, 1242 (1957).

17) In this reaction, the purity of the ylide and reaction temperature were very important. When crude ylide was used, unexpected side reactions due to both lowering of the $E, Z$-stereoselectivity and elimination of $\mathrm{H}_{2} \mathrm{O}$ occurred concomitantly to give an $E, Z$-mixture of $\alpha, \beta$-unsaturated esters and diene esters. The reaction proceeded quite rapidly at a higher temperature in refluxing benzene, but complex reaction products were obtained.

18) The $E, Z$-configuration was determined from the chemical shifts in the NMR spectra $(E, 6.83 ; Z, 6.73)$.

19) The di-DMPM product was also obtained in $14 \%$ yield, but its secondary DMPM group, not the primary one, was selectively deprotected by the treatment with DDQ at $-20{ }^{\circ} \mathrm{C}^{6)}$ to give 13 in $70 \%$ yield. Therefore, the combined yield of 13 was $c a .80 \%$.

20) Other reducing agents gave only unsatisfactory results. The reduction with $\mathrm{LiAlH}_{4}$ or (iso-Bu) ${ }_{2} \mathrm{AlH}(\mathrm{DIBAH})$ was incomplete due to formation of insoluble precipitates. Because of their fairly strong basicity, $\mathrm{NaBH}_{4}$ and $\mathrm{NaAlH}_{2}\left(\mathrm{OC}_{2} \mathrm{H}_{4} \mathrm{OCH}_{3}\right)_{2}$ (Red-Al) brought about epimerization of the $\mathrm{C}-8$ methyl group.

21) A. J. Mancuso, S.-L. Juang, and D. Swern, J. Org. Chem., 43, 2480 (1978).

22) E. J. Corey and G. T. Kwiatkowski, J. Am. Chem. Soc., 88, 5654 (1966).

23) E. J. Corey and G. Schmidt, Tetrahedron Lett., 1977, 399.

24) The expected product (16) was not obtained by usual Swern oxidation. Oxidation with pyridinium chlorochromate $(\mathrm{PCC})^{25}$ caused a concomitant oxidation at the benzylic position of the DMPM group to give a 3,4dimethoxybenzoate.

25) E. J. Corey and J. W. Suggs, Tetrahedron Lett., 1975, 2647.

26) R. U. Remieux and E. von Rudloff, Can, J. Chem., 33, 1701, 1710 (1955).

27) a) S. Masamune, Y. Hayase, W. Schilling, W. K. Chan, and G. S. Bates, J. Am. Chem. Soc., 99, 6756 (1977); b) H. Gerlach and T. Thalmann, Helv. Chim. Acta, 57, 2661 (1974); c) T. Mukaiyama, R. Matsuda, and M. Suzuki, Tetrahedron Lett., 1970, 1901; d) T. Mukaiyama, R. Matsuda, and H. Murayama, Bull. Chem. Soc. Jpn., 43, 1271 (1970); e) E. J. Corey, D. J. Brunelle, and P. J. Stork, Tetrahedron Lett., 1976, 3405; f) E. J. Corey and K. C. Nicolaou, J. Am. Chem. Soc., 96, 5614 (1974); g) J. Inanaga, K. Hirata, H. Saeki, T. Katsuki, and M. Yamaguchi, Bull. Chem. Soc. Jpn., 52, 1989 (1979).

28) P. A. Aristoff, J. Org. Chem., 46, 1954 (1981).

29) a) P. A. Grieco, J. Inanaga, and N.-H. Lin, J. Org. Chem., 48, 892 (1983); b) H. Matsubara, K. Miyano, A. Nakagawa, and S. Ohmura, Chem. Pharm. Bull., 30, 97 (1982).

30) The synthetic tylonolide (1) was a $3.3: 1$ isomeric mixture with respect to the hemiacetal position. Tylonolide derived from natural tylosin by Grieco's method $\mathrm{A}^{29 a)}$ was a $6: 1$ mixture (fine needles, $\mathrm{mp} 155-156^{\circ} \mathrm{C}$, after three recrystallizations from acetone-ether-hexane; lit. ${ }^{29 a} \mathrm{mp} 102.5-103.5^{\circ} \mathrm{C}$ or $157.5-158.5^{\circ} \mathrm{C}$ ). When its $\mathrm{CDCl}_{3}$ solution was allowed to stand in an NMR tube at room temperature, isomerization (probably catalyzed by a trace of acid in the solvent) occurred to give a $3: 1$ mixture. 